\title{
Asymptotics of large bound states of localized structures.
}

\author{
G. Kozyreff ${ }^{1}$ and S.J. Chapman ${ }^{2}$ \\ ${ }^{1}$ Optique Nonlinéaire Théorique, Université Libre de Bruxelles, \\ C.P. 231, Campus Plaine, B-1050 Bruxelles (Belgium) \\ ${ }^{2}$ OCIAM, Mathematical Institute, 24-29 St Giles's, Oxford OX13LB (UK)
}

(Dated: October 2, 2006)

\begin{abstract}
We analyze stationary fronts connecting uniform and periodic states emerging from a patternforming instability. The size of the resulting periodic domains cannot be predicted with weakly nonlinear methods. We show that what determine this size are exponentially small (but exponentially growing in space) terms. These can only be computed by going beyond all orders of the usual multiple-scale expansion. We apply the method to the Swift-Hohenberg equation and derive analytically a snaking bifurcation curve. At each fold of this bifurcation curve, a new pair of peaks is added to the periodic domain, which can thus be seen as a bound state of localized structures. Such scenarios have been reported with optical localized structures in nonlinear cavities and localized buckling.
\end{abstract}

In spatially extended dynamical systems featuring subcritical Turing instabilities, there generally exists a parameter value in the vicinity of which stable stationary fronts separate regions in space where the solution is either uniform or spatially oscillating. Owing to the wide variety of patterns that can result, this so-called Maxwell point has received much attention. Examples of applications are found in hydrodynamic [1], mechanical [2], vegetation [3] or nonlinear optical problems $[4,5]$. A particularly dramatic feature associated to this situation is a snaking bifurcation diagram that is composed of an infinite succession of folds $[2,5]$. Each fold signals the appearance of a new pair of peaks in the patterned domain, so that the latter can be viewed as a bound state of localized structures [6]. Moreover, this curve delimits the "pinning range" of parameters where localized structures can be found, which is a useful piece of information if these are to be used for data processing [7]. The phenomenon is robust and has been given a geometrical interpretation in the frame of dynamical system theory $[8,9]$. However a key question that has remained unanswered so far is: what determines the size of the patterned state, or equivalently, how to construct such a bifurcation curve. A crucial observation in this respect is that the standard multiple-scale analysis, which separates the slow spatial scale of the front from that of the fast-oscillating underlying pattern, is insufficient to resolve this problem [1]. Indeed, this procedure misses the exponentially small "non-adiabatic" effects that couple the slow and fast scales. In an attempt to compensate for this shortcoming, several authors have modified the multiple-scale analysis and did obtain some exponentially small terms $[10,11]$. Although some insight can be gained this way, their method is inconsistent in that the envelope, which is supposed to depend only on the slow scale, is proposed to satisfy an equation where the fast scale appears explicitly. This introduces the non-adiabatic effects after a few orders, whereas in fact they appear beyond all orders of the multiple-scale analysis.
The purpose of this letter is to show how to compute these effects and to demonstrate their physical relevance by constructing the snaking bifurcation diagram. We note that beyond-all-order techniques have been applied in a few instances to multiple-scale problems: gravitycapillary solitary waves [12], oscillating shock solutions of the Kuramoto-Sivashinsky equation [13, 14] and strut buckling on an elastic foundation [15]. The last analysis is close in spirit to the present one but breaks down near the Maxwell point. Let us also mention the recent alternative approach combining asymptotic and geometrical arguments in [16], which was successfully applied to a snaking bifurcation curve involving a saddle-point and a focus in phase space.

Presently, we analyze the Swift-Hohenberg equation

$$
\frac{\partial E}{\partial t}=\mathcal{Y}+\mathcal{C} E-E^{3}-\left(1+\partial_{x}^{2}\right)^{2} E
$$

relevant to the study of many pattern-forming systems [17]. For example, in nascent optical bistability, $E, \mathcal{Y}$, and $\mathcal{C}$ respectively denote deviations of the cavity field amplitude, the injected field amplitude, and the cooperativity parameter from the onset of bistability [4]. The unit length is set by the cavity detuning and the diffraction coefficient.

The homogeneous steady state $E_{h g}$ satisfies $\mathcal{Y}=E_{h g}^{3}+$ $E_{h g}-\mathcal{C} E_{h g}$ and there is a subcritical Turing bifurcation with unit wave number when $3 E_{h g}^{2}=\mathcal{C}$. In the following, instead of $\mathcal{Y}$ and $\mathcal{C}$, we will use the equivalent set of parameters $E_{h g}$ and $\varepsilon \ll 1$ defined through

$$
\mathcal{C}=3 E_{h g}^{2}-\varepsilon^{4}, \quad E(x, t)=E_{h g}+\varepsilon f(x) .
$$

Hence, $\varepsilon$ sets the order of magnitude of the oscillation at the Maxwell point. The scaling above is typical for stable branches of periodic pattern emerging from a subcritical bifurcation [17]. With this change of variable, the SwiftHohenberg equation becomes

$$
\left(1+\partial_{x}^{2}\right)^{2} f+\varepsilon^{4} f+3 \varepsilon E_{h g} f^{2}+\varepsilon^{2} f^{3}=0 .
$$


We want to study (2) for values of $E_{h g}$ such that homogeneous and periodic solutions can be separated by stable stationary fronts. To this end, we introduce the slow scale $X=\varepsilon^{2} x$ and look for a solution of the form

$$
\begin{aligned}
f(x) & =\sum_{n=0}^{N} \varepsilon^{n} f_{n}(x, X)+\delta f(x, X, \varepsilon), \\
E_{h g} & =E_{0}+\varepsilon^{2} E_{2}+\varepsilon^{4} E_{4}+\ldots+\delta E .
\end{aligned}
$$

The power series in (3) being generally divergent, it is truncated after $N$ terms and we denote the remainder by $\delta f$. On the other hand, the power series in (4) approximates the Maxwell point, while $\delta E$ is a small deviation from it. Substituting (3) and (4) in (2), one obtains a set of linear differential problems at each order in $\varepsilon$, of which the first few are solved in the usual way: first, setting $\varepsilon=0, f_{0}$ is seen to be a harmonic function of $x$ with period $2 \pi$ and slow-varying complex amplitude, denoted by $f_{0,1}(X)$. Secondly, a solvability condition at $O\left(\varepsilon^{2}\right)$ is that $E_{0}=\sqrt{3 / 38}$. Finally, at $O\left(\varepsilon^{4}\right)$, a new solvability condition yields the amplitude equation

$f_{0,1}^{\prime \prime}+\frac{4 i\left|f_{0,1}\right|^{2}}{19} f_{0,1}^{\prime}=f_{0,1}\left(\frac{1}{4}-\sqrt{\frac{57}{2}} E_{2}\left|f_{0,1}\right|^{2}+\frac{2205}{361}\left|f_{0,1}\right|^{4}\right)$

for $f_{0,1}(X)$. This amplitude equation is found to have front solutions for $E_{2}=\sqrt{5872 / 20577} \approx 0.534$ and hence, adopting this value, the leading order solution is

$$
f_{0}(\bar{x}, X)=\sqrt{19 \beta / 2} \frac{e^{X / 2}}{\left(1+e^{X}\right)^{1 / 2+i \beta / 2}} e^{i \bar{x}}+\text { c.c. },
$$

where $\beta=1 / \sqrt{734}, \bar{x}=x+\varphi$, and c.c. means "complex conjugate". In this expression, $\varphi$ denotes the phase of the fast oscillations relative to the slow scale and is unknown at this stage. This solution describes the onset of oscillation but doesn't predict return to the homogenous state as $X \rightarrow \infty$. One could expect to find this piece of information by computing higher order terms in (3) and introducing slower space scales. However, no matter how far one proceeds in this way, no sign of the amplitude of oscillation going back to zero for large $X$ ever shows up. This means that the terms responsible for this down-switching lie beyond all orders of the multiple-scale expansion.

In (3) the magnitude of the terms $\varepsilon^{n} f_{n}$ first decreases with $n$ but eventually increases, thus making the series divergent. In order to capture exponentially small terms, we truncate the sum in (3) near its smallest term and compute the remainder $\delta f$. Indeed, it is expected that, for large $n$, the magnitude of $f_{n}$ will be given roughly by $\Gamma(n / 2+\alpha+1)$ for some constant $\alpha$ [18]. Hence, using Stirling's formula, $\varepsilon^{n} f_{n}=O\left(\varepsilon^{n}(n / 2)^{n / 2+\alpha+1 / 2} e^{-n / 2}\right)$ and the least term, obtained for $N / 2 \approx \varepsilon^{-2}$, is thus $O\left(\varepsilon^{-2 \alpha-1} e^{-1 / \varepsilon^{2}}\right)$. This makes the magnitude of the remainder in (3) of the right order to study non-adiabatic effects. Recently, there has been considerable progress in the mathematical literature on the optimal choice of normal-form truncation [19].

Assuming then that we have determined $f_{0}, f_{1}, \ldots f_{N}$, the equation for the remainder is

$$
\begin{gathered}
\left(1+\left(\partial_{x}+\varepsilon^{2} \partial_{X}\right)^{2}\right)^{2} \delta f+\varepsilon^{4} \delta f+6 \varepsilon\left(E_{0}+\ldots\right) \\
\times\left(f_{0}+\varepsilon f_{1}+\ldots\right) \delta f+3 \varepsilon^{2}\left(f_{0}^{2}+\ldots\right) \delta f \\
=-3 \varepsilon \delta E\left(f_{0}^{2}+2 \varepsilon f_{0} f_{1}+\ldots\right)+r h s_{N} .
\end{gathered}
$$

Since $\delta f$ is exponentially small, one can safely neglect all nonlinear terms in $\delta f$ in $(7)$. The right hand side is split into two parts. The first one is due to the deviation from the Maxwell point and can again be treated by the multiple-scale method. This produces the contribution $\delta f^{E}$ in $\delta f$, given by

$$
\frac{i \ell_{1} \varepsilon^{-2} \delta E e^{3 X / 2} e^{i \bar{x}}}{\left(1+e^{X}\right)^{3 / 2+i \beta / 2}}\left[e^{X}(\beta+i)+\beta+2 i+\text { e.d.t. }\right]+\text { c.c. },
$$

where $\ell_{1}=\sqrt{114}(19 \beta / 8)^{3 / 2}$ and "e.d.t." stands for exponentially decaying terms as $X \rightarrow \infty$. On the other hand, $r h s_{N}$ is the remainder obtained after substituting the truncated multiple-scales expansion for $f$ into the equation. It depends on the large- $n$ terms of this expansion, and its determination makes up the majority of the effort behind this work. Here we only sketch the calculation.

Let us first observe that the leading order solution (6) has the two simple poles $X= \pm i \pi$. Such singularities are known since [20] to be intimately related to the existence of exponentially small terms in $f$. More specifically, these terms will be shown to emerge from a Stokes line that joins the two singularities.

One can decompose the $n^{\text {th }}$ term in (3) as $\sum f_{n, k}(X) \exp i k \bar{x}$, with $k=-n-1, \ldots, n+1$. Equation (2) then yields a recurrent set of equations for the functions $f_{n, k}(X)$, which can be set up as an eigenvalue problem in the limit as $n \rightarrow \infty$. In the present case, it quickly emerges that only harmonics for which $n+k$ is odd are nonzero. For given $n \gg 1, n$ odd, the eigenvector that is relevant to our discussion is then found to be

$$
\left(\begin{array}{c}
f_{n, 0} \\
f_{n, 2}
\end{array}\right)=\ell_{2} \frac{\kappa^{n} \Gamma\left(\frac{n}{2}+\alpha+1\right)}{(i \pi-X)^{n / 2+\alpha+1}}\left(\begin{array}{c}
F_{0}(X)+O(1 / n) \\
F_{2}(X)+O(1 / n)
\end{array}\right),
$$

all other components $f_{n, k}$ with the same $n$ being at least $O(1 / \sqrt{n})$ smaller. In this expression, $\kappa=\sqrt{i}$ is the associated eigenvalue, $\alpha=2+i \beta / 2, \ell_{2}$ is a constant, and

$$
\begin{aligned}
F_{1 \pm 1}(X)= & \frac{e^{3 X / 2}}{\left(1+e^{X}\right)^{3 / 2 \pm i \beta / 2}}\left[6+e^{X} \pm i \beta\right. \\
& \left.\times\left(4-4 X+4 i \pi-e^{X}\right)+\text { e.d.t. }\right]
\end{aligned}
$$

This was derived using $1 / \sqrt{n}$ as a small parameter in the recurrence equations. The value of $\ell_{2}$ can only be 
determined numerically, either by solving the recurrence equations exactly all the way up to large values of $n$, or by solving (2) numerically for an appropriate set of parameter values. Let us remark that (9) does not represent the full solution $\left\{f_{n, k}\right\}$ for large $n$. In particular, there is another eigenvector, associated to $\kappa=\sqrt{-i}$, and for which the dominant elements are $f_{n, 0}$ and $f_{n,-2}$. However, its contribution to $r h s_{N}$ will not affect our result at leading order, so we will simply ignore it in the present discussion. We also draw attention to the special role played by $X=i \pi$ in (9). Actually, there is a complementary set of eigenvectors associated to $X=-i \pi$ such that $f_{n}(x, X)$ is real for real values of $X$. These will be taken into account at the end of this development by requiring that the remainder $\delta f$ be real as well.

Using (9), we now compute $r h s_{N}$ in the vicinity of the Stokes line, which joins $X=i \pi$ and $X=-i \pi$. To this end, we set $X=i \pi-i r \exp i \varepsilon \theta$ with $r$ fixed between 0 and $2 \pi$ and $\theta$ variable. The latter corresponds to the Stokes variable in Berry's treatment of Stokes's discontinuities [18]. With this parameterization, $X$ is on the Stokes line when $\theta=0$. The large- $n$ terms of $\left\{f_{n, k}\right\}$ are then found to produce

$$
4 i \ell_{2} \varepsilon \theta\left(F_{0}+F_{2} e^{2 i \bar{x}}\right) \frac{(\varepsilon \kappa)^{N+2} \Gamma(N / 2+\alpha+2)}{(i \pi-X)^{N / 2+\alpha+2}}
$$

in $r h s_{N}$ and the key observation [14] is that, at optimal truncation, where $N / 2+\alpha+1 \approx r / \varepsilon^{2}$, the fraction above is asymptotic to

$$
i \sqrt{\frac{2 \pi}{r}} \varepsilon^{-5} \exp \left(\frac{\beta \pi}{4}-i \beta \ln \varepsilon-\frac{\pi}{\varepsilon^{2}}-\frac{r \theta^{2}}{2}-i \frac{X}{\varepsilon^{2}}\right) .
$$

This means that the fraction in (11) is locally equivalent to the fast oscillating factor $\exp \left(-i X / \varepsilon^{2}\right)=\exp (-i x)$ and this turns $r h s_{N}$ into a resonant driving term in (7). This is only true very close to the Stokes line, as attested by the $\exp \left(-r \theta^{2} / 2\right)$ factor. Because of this fast variation relative to the $X$-scale, we must solve (7) locally using $\theta$ instead of $X$ as the slow scale. In doing so, we find that $r h s_{N}$ produces the particular solution

$$
\delta f^{+}=\varepsilon^{-2} \gamma \ell_{2}^{\prime} \frac{F_{0} e^{-i \bar{x}}+F_{2} e^{i \bar{x}}}{2} e^{i \varphi-i \beta \ln \varepsilon} \int_{-\infty}^{\theta} \frac{e^{-r v^{2} / 2}}{\sqrt{2 \pi / r}} \mathrm{~d} v
$$

in $\delta f$, where $\gamma=\varepsilon^{-4} \exp \left(-\pi / \varepsilon^{2}\right)$ and $\ell_{2}^{\prime}=$ $4 \pi \ell_{2} \exp (\beta \pi / 4)$. In this expression, the integral factor vanishes as $\theta \rightarrow-\infty$ (corresponding to $X=i(\pi-r)+0^{-}$) and equals one as $\theta \rightarrow \infty$ (corresponding to $X=$ $\left.i(\pi-r)+0^{+}\right)$. Thus $\delta f^{+}$is "switched on" upon crossing the Stokes line towards positive values of $X$ [18]. Finally, having obtained $\delta f^{+}$, the complementary contribution $\delta f^{-}$associated to the pole $X=-i \pi$ makes $\delta f$ real on the real- $X$ axis. Hence, for $X<0$,

$$
\delta f=\delta f^{E},
$$

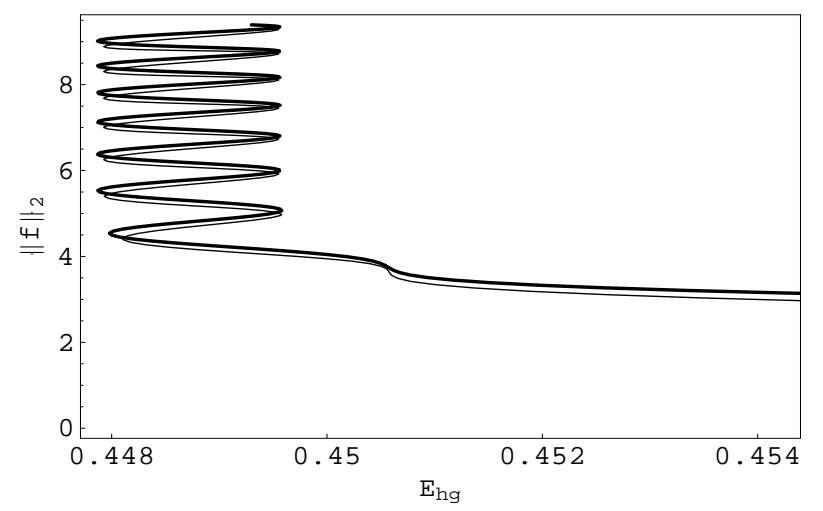

FIG. 1: Bifurcation diagram for $\varepsilon=0.55$. Thick line: analytical formula. Thin line: numerical simulation.

but for $X>0$,

$$
\delta f=\delta f^{E}+\varepsilon^{-2} \gamma \operatorname{Re}\left[\ell_{2}^{\prime}\left(F_{0} e^{-i \bar{x}}+F_{2} e^{i \bar{x}}\right) e^{i \varphi-i \beta \ln \varepsilon}\right] .
$$

We now have all the necessary information to construct a finite spatial domain of oscillation. Indeed, $\delta f$, although exponentially small, is an exponentially increasing function of $X$ and this ultimately leads to a downswitching front. Assembling the various pieces of solution we have obtained thus far, we construct a finite periodic domain of size $d \gg 1$. On the left of the domain, the solution is given by the approximate formula

$$
f\left(x, X ; \varphi_{L}\right) \approx f_{0}\left(x+\varphi_{L}, X\right)+\delta f\left(x, X ; \varphi_{L}\right),
$$

while, by symmetry, the right side is described by the down-switching front $f\left(d-x, \varepsilon^{2} d-X ; \varphi_{R}\right)$. From (6), the oscillations in the left part are controlled by the exponential factor $\exp i\left(x+\varphi_{L}-\beta X / 2\right)$ as $X \rightarrow \infty$, while in the right part, the corresponding factor is $\exp i\left(x-d-\varphi_{R}-\beta X / 2+\varepsilon^{2} \beta d / 2\right)$ as $\varepsilon^{2} d-X \rightarrow \infty$. In order for these oscillations to smoothly join, we thus have to impose

$$
\varphi_{L}=-\varphi_{R}-d+\varepsilon^{2} \beta d / 2+2 m \pi,
$$

where $m$ is an arbitrary integer. Considering next slow-varying amplitudes, the exponentially growing terms in $\delta f\left(x, X, \varphi_{L}\right)$ [see (8) and (10)] must match the exponential start of the down-switching in $f_{0}\left(d-x+\varphi_{R}, \varepsilon^{2} d-X\right)$. This leads to $\varphi_{R}=\varphi_{L}$ and

$$
\delta E= \pm \gamma c_{1} \cos \left(\frac{2-\varepsilon^{2} \beta}{4} d+\beta \ln \varepsilon+c_{2}\right)+2 \varepsilon^{2} E_{2} e^{-\varepsilon^{2} d} .
$$

The latter is the bifurcation equation between the bifurcation parameter $\delta E$ and the front separation $d$. The constants $c_{1}$ and $c_{2}$ are proportional to $\left|\ell_{2}^{\prime}\right|$ and $\arg \ell_{2}^{\prime}$ and hence must be determined numerically. This bifurcation equation actually defines two interlaced snaking bifurcation curves; one corresponds to an even number of peaks, the other to an odd number of peaks. 


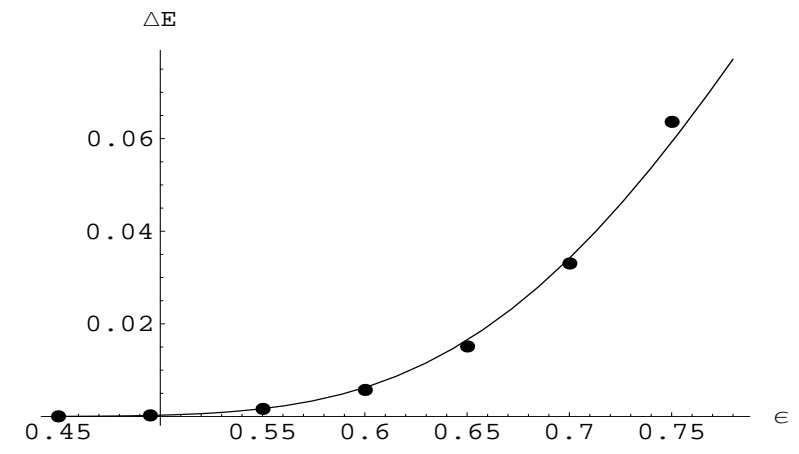

FIG. 2: Amplitude of the snaking oscillations (pinning range) in Fig. 1 as a function of $\varepsilon$. Dots: numerical; line: analytical.

We checked the prediction from our formula by computing numerically the bifurcation curve with the continuation package AUTO [21] and found excellent agreement, even for moderately small values of $\varepsilon$ and moderately large localized structure size $d$. In Fig. 1, we plot the numerically and asymptotically determined L2-norm of $f$. To compute the latter, we used a composite development based on $f(x, X, \varphi)$ and $f\left(d-x, \varepsilon^{2} d-X, \varphi\right)$ and included the $O(\varepsilon)$ terms from the weakly nonlinear analysis. From (13) and (4), the pinning range $\Delta E$ of values of $E_{h g}$ where localized structures exist scales like $\gamma$. This is again fully confirmed, as shown in Fig. 2. The value $c_{1}$ can be determined graphically by plotting $\frac{1}{2} \Delta E \varepsilon^{4} \exp \pi / \varepsilon^{2}$. All the points in Fig. 2 then appear on an horizontal line of height $c_{1} \approx 2.439$. Next, $c_{2}=-0.5$ provides the best fit in Fig. 1 .

In conclusion, a consistent analysis beyond all orders of the weakly nonlinear analysis elucidates the interaction between the slow and fast scales in pattern-forming instabilities. This interaction takes places near the Stokes line that joins the two first poles of the slow-varying front and leads to the emergence of exponentially small terms in the solution. These eventually dominate and lead to a second, down-switching front, thus allowing us to analyze localized structures asymptotically. Furthermore the analysis can easily be extended to the study of a larger number of fronts, to dynamical fronts, and the effect of noise, whereby $d$ becomes an exponentially slow function of time. Because the analysis presented is local, it bears the same generality as any bifurcation analysis and is not restricted to the particular equation that we have studied here. In particular, Eq. (1) can be deduced in some limit from the diffraction-type Ginzburg-Landau equation with saturable nonlinearity [22]. In principle, it could be applied to any subcritical Turing instability near the Maxwell point.

This research was supported in part by the Fonds National de la Recherche Scientifique (FNRS, Belgium) and the Interuniversity Attraction Pole Program of the Belgian government. We thank Michel Nizette and Mustapha Tlidi for useful comments on the manuscript.

[1] Y. Pomeau, Physica D 23, 3 (1986).

[2] G. W. Hunt et al., Nonlinear Dynamics 21, 3 (2000).

[3] O. Lejeune, M. Tlidi, and P. Couteron, Phys. Rev. E 66, 010901(R) (2002).

[4] M. Tlidi, P. Mandel, and R. Lefever, Phys. Rev. Lett. 73, 640 (1994).

[5] J. M. McSloy et al., Phys. Rev. E 66, 46606 (2002).

[6] V. B. Taranenko et al., Phys. Rev. A 61, 63818 (2000).

[7] S. Barland et al., Nature 419, 699 (2002).

[8] G. W. Hunt, G. J. Lord, and A. R. Champneys, Comput. Methods Appl. Mech. Engrg. 170, 239 (1999).

[9] P. Coullet, C. Riera, and C. Tresser, Phys. Rev. Lett. 84, 3069 (2000).

[10] D. Bensimon, B. I. Shraiman, and V. Croquette, Phys. Rev. A 38, 5461 (1988).

[11] M. G. Clerc, C. Falcon, and E. Tirapegui, Phys. Rev. Lett. 94, 148302 (2005).

[12] T. S. Yang and T. R. Akylas, J. Fluid Mech. 330, 215 (1997).

[13] T. S. Yang, Physica D 110, 25 (1997).

[14] K. L. Adams, J. R. King, and R. H. Tew, J. Eng. Math. 45, 197 (2003).

[15] M. K. Wadee, C. D. Coman, and A. P. Bassom, Physica D 163, 26 (2002).

[16] J. Knobloch and T. Wagenknecht, Physica D 206, 82 (2005).

[17] M. C. Cross and P. C. Hohenberg, Rev. Mod. Phys. 65, 851 (1993).

[18] M. V. Berry, Proc. R. Soc. A 422, 7 (1988).

[19] G. Ioos and E. Lombardi, C. R. Math. Acad. Sci. Paris 339, 831 (2004); J. Diff. Eq. 212, 1 (2005).

[20] H. Segur and M. D. Kruskal, Phys. Rev. Lett. 58, 747 (1987).

[21] available at http://cmvl.cs.concordia.ca/auto/.

[22] M. L. Berre, E. Ressayre, and A. Tallet, Quantum Semiclass. Opt. 7, 1 (1995). 